\title{
Fun, communication and dependability: extending the concept of usability
}

\author{
Andrew F. Monk
}

Department of Psychology,

University of York, York YO1 5DD, U.K.

Email:A.Monk@psych.york.ac.uk

Designing information and technology products for the home has drawn attention to the narrowness of traditional conceptions of usability. Design methods and guidelines were developed in work contexts and so are mainly concerned with ease-of-learning, low level ease-of-use and task fit. These are all issues in the home but there are other important components of usability. How do we make the product enjoyable to use? How do we design for effective humanhuman communication? How doe we make these systems dependable? The paper illustrates these issues through two design problems, the Virtual Pub and Mavis' smart home. Drawing on the history of HCI, suggestions are made about how these new conceptions of usability could be further developed for design for the home, and more widely.

Keywords: usability, methodologies, guidelines, fun, enjoyment, effective communication, dependability.

\section{Technology in the home - a stimulus for change}

The technology we use in our homes is becoming increasingly sophisticated. Entertainment systems such as multi-channel interactive TV require complex interfaces. Future visions of ubiquitous computing in the home involve networked appliances linked to sensors and effectors that react automatically to our behaviour. The manufacturers and vendors of this technology are aware that human factors are crucial in determining uptake. We are paid to use the technology provided at work. If it breaks down regularly or is tedious to use we will put up with it. Attitudes to 
technology we have bought ourselves are somewhat different. We need it to be reliable, attractive and enjoyable to use.

This paper will argue that conventional approaches to usability are too narrowly focussed to properly inform the design of technology for the home. It will suggest some issues that need to be addressed in order to broaden the concept of usability. These are: enjoyment (fun), effective communication and dependability. Designing for the home has highlighted these issues but they are also important in work and educational contexts. In this sense designing for the home context is a stimulus for a more wide ranging change in the way we conceptualise usability.

\section{Usability as ease-of-learning, low level ease-of-use and task fit}

What do I mean by conventional approaches to usability? To design a product to support someone's work in the office one needs to achieve usability through ease-of-learning, low level ease-of-use and task fit. HCI research has been very successful in this area and there is considerable agreement on how to do this. For the first two ease-of-learning and low level ease-of-use one may rely on standards and guidelines. ISO 9241 specifies how and when menus, forms and other dialogue styles should be used. International standards have to be agreed by numerous committees and have the weight of law behind them. However, the less formal style guides have probably been more influential. These prescribe how the interface should work for a particular operating system. The knowledge they contain has been incorporated into the tools that are used to create software. Thus, a designer using a software development tool such as Visual Basic will find it very hard to disobey the Windows Microsoft Windows style guide (Microsoft, 1995) even if they have never read it. This prevents the designer from developing idiosyncratic interfaces that do not behave in the way users expect. At the very least, by enforcing a degree of consistency, style guides ensure ease-of-learning. When a user learns to do something in one context, that knowledge will transfer positively to new contexts. This is important as even small inconsistencies can result in negative transfer. It is a common experience to upgrade software only to find it extremely difficult to suppress ingrained habits learned with the old system. In fact, the Windows style guide and those that came before it draw on the results of years of painstaking empirical and analytic work from the HCI research community and they do guarantee low level ease-of-use. In this way I have argued (Monk, 2000) that Visual Basic is a theory of HCI. Software tools encapsulate style guides, and style guides encapsulate the research.

When designing a product for use in the office then, ease-of-learning and low level ease-of-use are achieved by specifying the product of design, that is how the interface should appear and behave. Task fit is achieved by specifying the process of design, how the designer should behave. Here again there is considerable agreement and an international standard, ISO 13407. There are also text books (e.g., Dix, Finlay, Abowd \& Beale, 1998) and proprietary methodologies such as Contextual Inquiry (Holtzblatt \& Jones, 1993) and Monk's Lightweight Techniques (Monk, 1998). There are 
certain common elements to all these processes and these are illustrated in Table 1.

Understanding the work context

Methods: focus groups, interviews, observation

Representations: the rich picture

Understanding the work

Methods: focus groups, interviews, observation

Representations: Hierarchical Task Analysis

(HTA), scenarios

Testing a top level design against your understanding of the work

Methods: Scenario walkthrough, Cognitive Walk Through

Representations: Story boards, dialogue

modelling

User testing of more detailed prototypes

Methods: Usability Labs., Cooperative

Evaluation

Representations: Paper prototypes, simulations

Table 1. Common processes in user centred

design.

The user-centred design process starts by obtaining an understanding of the context for the work. In broad terms, how does the work get done? Who are the stakeholders in this system and what are their concerns? The methods used to elicit this information, interviews and observation, are unremarkable, though they require a great deal of skill to carry out well. What the various proprietary methodologies prescribe are representations, ways of recording and thinking about the information obtained through interviews and observation. The Rich Picture (Checkland \& Scholes, 1990; Monk, 1998) is one such representation. It consists of a cartoon style depiction of the key stakeholders, their concerns and how the work passes between them. It is common to give each stakeholder a "thought bubble" expressing his or her main concerns about the work. Writing down the findings in this way focuses the analysis and allows the analyst to reason about the completeness of her understanding. It can also be taken back to the analyst's informants to check the understanding obtained. The rich picture, like all the other representations listed in Table 1, is also a valuable tool for communicating with designers.

Table 1 also lists some of the representations that have been proposed for recording and reasoning about the work done by a particular operator. A high level design can be tested against this understanding. Finally, a detailed prototype of the interface can be built and refined by user testing.

So, what parts of this conventional view of usability can be used to design for the home? The standards and style guides used to achieve easeof-learning and low level ease-of-use for software for the office will be of 
some use. Unfortunately, most are for graphical interfaces operated with a mouse and a keyboard. In the home controls and displays tend to be smaller. We have to return to basic principles of interaction design and develop style guides and software tools that reinforce those guidelines for the new forms of interaction needed in the home.

The design methodologies, and associated representations, specified to achieve task fit will also be applicable at some level. There are tasks to be done in the home. Setting the video recorder or doing the laundry are work and are amenable to these methods. But, where is the task in actually watching the TV or talking on the telephone?

In the domestic environment user concerns go well beyond task fit. To take another example, various products have been devised for the elderly that make allowances for fading eyesight and poor manual dexterity. Many of these apparently useful products have failed simply because they are ugly and stigmatising. We need to go back to basics, to identify new usability concepts, parallel to ease-of-learning, ease-of-use and task fit, but with a broader reach. In the sections that follow I will speculate about some such concepts under the headings: enjoyment, effective communication, and dependability. Note, the words "fun" and "enjoyment" are used interchangeably in this paper. "Fun" makes for an arresting title but "enjoyment" may be more useful in a taxonomy of usability as it implies a less specific user experience.

\section{Enjoyment}

To make this discussion more concrete, consider the following design problem. Broadband Internet makes group conversations using voice an alternative to telephone conferencing that may be sufficiently inexpensive to be used for leisure purposes. In addition, graphical support for this leisure experience could be provided over the internet in the form of pictures and text. How would you design an inexpensive terminal, let us call it "the virtual pub", to make it possible for geographically distributed friends to get together without anyone having to travel?

Conventional concepts of usability can be applied to the problem of connecting the friends up and then operating the other features one might want to include. But what are these features, and what are they there to achieve? One requirement is clearly that the conversations should be enjoyable or fun and so the terminal should include features that facilitate fun conversations. This is equivalent to identifying ease-of-learning as a requirement for office products. On its own it is not worth a lot. We need to understand the concept of enjoyment further before we can guide design. Let us examine the history of HCI to see how that might be done.

Several years of empirical and analytic work were required to identify the properties of a user interface necessary for ease-of learning. Different forms of consistency were identified, for example, and these in turn lead to the formation of the guidelines that eventually gave rise to the style guides used today. The empirical work needed to gain this understanding followed the psychological paradigm of experimentation. Different user interfaces were constructed and compared by having users work with them. Measures pertaining to particular criteria to do with ease-of-learning, e.g., learning 
time, were devised to make these comparisons. The analytic work similarly drew on ethnographic studies or simply the common experience of reader and author with different systems to find examples that could identify features of an interface that lead to good or bad ease-of-learning.

Can we follow this process with the high level concept of "enjoyment". The key is to break enjoyment down into components that are sufficiently specific that we can agree when they are there or not, and if possible, how to measure them. There is some existing work from outside of HCI that can be drawn on here. In the psychological tradition, Csikszentmihalyi and colleagues (see for example Csikszentmihalyi \& Rathunde, 1993) have examined the conditions necessary for a "flow" experience. They interviewed people like rock climbers who spent large amounts of their time on difficult activities that have no apparent external motivation. An important common theme in the experiences of all these people was "flow". The activity takes them over shutting out all other concerns. In sport this experience has been described as being "in the zone". The conditions necessary to get a flow experience were: (i) an activity with clear goals and good feedback as to whether these goals are being achieved, and (ii) a close match between the difficulty of the task and the ability of the user. Computer games have these characteristics, scores provide goals and feedback. Difficulty is matched to ability through the levels within the game.

More mundanely, we have all had the experience of getting so involved in a task that we shut out everything else and it becomes an end in itself. This is rather different from the peak experience described by Csikszentmihalyi's informants and may be better described as "high engagement" rather than flow. Csikszentmihalyi and Rathunde (1993) developed a short list of questions that can be used repeatedly to assess minute-to-minute changes in flow, rather as the NASA TLX measures minute-to-minute changes in workload. Csikszentmihalyi and Rathunde's scale can also be used to measure engagement.

Flow and engagement are concepts that apply to the individual. Our design problem, the Virtual Pub, is about enjoyment in the form of engagement as a group. A sociological view of high engagement as fun is provided by Goffman (1961) through his concept of a focussed gathering.

'For the participants, this involves: a single and cognitive focus of attention; a mutual and preferential openness to verbal communication; a heightened mutual relevance of acts; an eye-to-eye ecological huddle that maximizes each participant's opportunity to perceive the other participants' monitoring of him' (Goffman, 1961:18).

The transcript in Table 2 is from a yet unpublished transcript by Darren Reed that we think exhibits these characteristics. It comes from a telephone conference between a facilitator and three older women. The "Freindship Links" telephone conference is one of a series organised by Hackney Burough Council for the benefit of isolated old people. For a large part of the recording the conversation is stilted, as Laura (the facilitator) goes around them one at a time trying to find some common interest. At the point where this extract starts she succeeds in generating smooth participation for the first time with a topic of favourite authors. Note how they are all jumping in with spontaneous contributions. Square parentheses 
indicate overlapping speech, and " $="$ at the end of one turn and the start of another indicates that the two utterances were "latched", i.e., one immediately followed the other with almost no pause between them. The amount of overlap and latching in this extract is much higher than elsewhere in the transcript. At last they are engaged in a common experience.

Laura: oh yes [ and which is what is your favourite author

Ida: $\quad$ ye

Ida: (0.2) (sniff) well i like er daniel steele=

Laura: $=$ oh yes yes

Ida: or catherine cookson=

Laura: =oh YEs yes dorothea and I [like catherine cookson

Dorothea:

${ }^{\circ}$ yeah $^{\circ}(($ cough $))$

Renie:

[thats mar favourit

Ida:

[( ) I like=

Laura: =an you as well renie $=$

Renie: =yeah=

Ida: = yes I like er ACHullee there as lot of (.) good books i like really,

Laura: $\mathrm{mm}$

Renie: the trouble is with er books once you've picked them up you dont want to put

Renie: them down [do you

Ida: $\quad\left[\mathrm{NO}: \mathrm{NO}^{\mathrm{N}}\right.$

Laura: [no::

Laura: [its ( )

Ida: [they are good yeah

Table 2. Extract form a transcript demonstrating a focussed gathering

To summarise this far, a high level of engagement as an individual or as a group would seem to be an important component of enjoyment and an important ingredient in many recreational activities. We can also identify some ways of measuring or detecting it, through rating scales or in conversations, that can be used in field studies and experiments. Using these measures, new features for the Virtual Pub could be evaluated by examining their ability to produce recreational experiences that exhibit a high level of engagement from the whole group. Analytic studies can proceed by making arguments about features that may induce or prevent high engagement. To take one example, a way of inducing engagement as a group would be for each participant to bring a "gift". This would take the form of a picture that would be displayed on all the Virtual Pub terminals. Participants would download their gifts to a website prior to the session and the software would cycle through the gifts so that each participant would get a turn at being the centre of attention for the group. There are existing photo sharing websites that already do something like this. 
One can think of other elements of enjoyment. Aesthetic attraction would seem to be important, also narrative completeness. Again, there are existing bodies of research that can be adapted to our purpose. Who knows, in the process of broadening the concept of usability HCI researchers may identify completely new components of fun and enjoyment.

\section{Effective communication}

Despite the implications of the phrase "human-computer interaction", a large part of the research within the discipline of HCI is concerned with electronic products that support human-human interaction. Enjoyment is a very new issue for HCI. Effective communication is an old one. Communication is studied under various headings, notably, "computer supported cooperative work", "electronically mediated communication" and "computer mediated communication". Hundreds of papers and books have been written on these topics. It is thus surprising that there has not been the same effort to encapsulate the results of this research in guidelines and design methodologies. The technological infrastructures for electronic communication depends on widespread agreement to adopt standard ways of doing things and international standards abound in this area. However, I am not aware of any international standards that prescribe user-centred guidelines on how text-based or voice communication should work, in the way that ISO 9241 prescribes how menus should work. Neither am I aware of an international standard prescribing a user centred methodology for the design of communication technology. There is also little in the way of agreed representations for reasoning about the problem.

Consider again the Virtual Pub. What features should we include to facilitate effective communication? To answer this question it is necessary to consider the nature of spoken conversation as a collaborative activity. Grice (1957) gives the following example. A couple are lying in bed. Anne says to Bill "What time is it?" Bill says "The milkman has just come". This reply only has meaning if Anne can assume that Bill is seeking to answer her question and knows that Anne knows when the milkman usually delivers the milk. As can be seen from Table 2, spoken language is poorly formed and disjointed in comparison to the sloppiest written language, and yet it is incredibly efficient. Clark (1996) explains how this efficiency arises through the collaborative obligations between the speaker and the hearer. For each utterance, the speaker and hearer are collaborating at a number of levels. At the most basic level, the listener is listening and the speaker is speaking. At a higher level, the speaker is designing what she says using her knowledge of the listener, and the listener is trying to understand given his knowledge of her knowledge. The listener has an obligation to signal whether he has understood and the speaker has an obligation to monitor these signals and repair potential "trouble" when she spots it.

The efficiency of spoken conversation is achieved, within the collaborative framework described above, using certain conversational devices described by Conversation Analysts (see for example Hutchby \& Wooffitt, 1998). This very influential branch of sociology is also the basis of Clark's account. I shall concentrate on just three conversational devices 
that I will dub, "interpersonal awareness", "fluent turn allocation" and "deixis".

First consider the concept of interpersonal awareness (Short, Williams \& Christie, 1976). A group of friends socialising in the same room are able to see each other, the environment they are in, and what they are doing in that environment. If one person slipped out to the toilet and another was taking a call on his mobile phone everyone would know. In the Virtual Pub this kind of awareness may be much harder to achieve. Another kind of awareness that may be absent in mediated communication is gaze awareness. Experiments at York (Monk \& Gale, 2002) have shown that we can accurately judge what someone is looking at in the immediate environment and that this knowledge can be used to make language more efficient. Again, unless the virtual pub gives you a view of all the participants and their surrounding environment, this will not be possible.

In one-to-one telephone conversations we have learned tricks to provide awareness. For example, if someone has a very long turn at talking, the other is obliged to make encouraging noises at the other end to prove they are still there. With a group conversation this may be harder to do. General awareness of who is attending in the Virtual Pub could be supported by low quality video with a wide angle view, for example, though this would not support full gaze awareness or eye contact.

Second, fluent turn allocation is similarly something that is hard when you cannot see the other person, and is even harder in a group. Conversation is structured as turns and Conversation Analysts have devoted a great deal of work to describing how a group conversation moves smoothly from one speaker to the next. When turn allocation is hard conversations become less fluent. The average turn size goes up and this impedes the rapid feedback necessary in the collaborative endeavour described above.

One important signal used in turn allocation is gaze and eye contact. An interesting potential solution to the problem of turn allocation has been provided by Vertegaal (1999). His GAZE system monitors the gaze of a participant using a camera and computer vision techniques. Instead of sending a video signal, which could be very expensive in a group conversation, one of a small number of alternative gaze positions is determined and used to control the graphical display of each participant. The display shows where each of the others is looking by using a few stored photos. So if Anne is looking at your photo on her display and Ben is looking at Anne's photo on his, your display will contain a photo of Anne looking straight ahead (at you) and Ben looking to the left (assuming Anne's photo is left of Ben's).

Third deixis simply means pointing with language. Whenever we say "that one" we are using deixis. Take the example of a meeting around a whiteboard. During the meeting various lists and diagrams will be put on the whiteboard. Later in the conversation these can provide a very powerful and efficient way of expressing oneself. Just by pointing, verbally or physically, to some item on the whiteboard one can reference a whole raft of previous talk that would take a long time to specify any other way. Something similar is happening during recreational conversations when we pass around a letter or a postcard, or a pack of holiday snaps. 
The importance of deixis suggests features such as virtual photograph albums. The idea of a graphical "gift", described in the discussion of enjoyment above, could also serve this purpose. Such a device would provide a commonly experienced object or field of reference so that deictic references to 'that' 'there' could be directed toward a common spatial context.

It is possible then to identify elements necessary for effective communication that one would want to support in the Virtual Pub. The examples given here are interpersonal awareness, fluent turn allocation and deixis. These are all specific enough and sufficiently well understood that we can make a priori arguments about the kinds of features which would facilitate them. It is also possible to identify them in a conversation and they can be used as criteria in experiments or field studies comparing alternative systems. The next step would seem to be some intensive analytic and empirical work to devise guidelines and methodologies for designers.

\section{Dependability}

Dependability has been a concern of Computer Scientists for many years (see for example Sommerville, 2000). In the office we are used to software that "crashes" on a regular basis. However, if you are building software to control a power station, or the control surfaces of an aircraft, you need to be able to prove that the software is very unlikely to fail or even to behave in an inappropriate manner. At York we are part of a large interdisciplinary project exploring how this work on "dependable systems" can be applied to socio-technical systems in general and the home in particular (see DIRC in references for website). To explain why this is important I need to develop another design problem.

One of the big opportunities provided by so-called "smart technologies" for the home is the possibility of enabling older and disabled people to remain independent and live in their own homes when they would otherwise have to move to some sort of institution. Imagine you are planning to install some technology in the home of an elderly woman, let us call her Mavis. She has severe arthritis and it has been decided to install motorised windows and doors with push button controls. These are linked through a wireless network and so we can also provide central locking. When she goes out, she can shut all open windows and lock both doors with one button press. She suffers from low blood pressure and could easily faint so we also install a personal fall monitor. This wearable device communicates with a call centre from which appropriate actions are initiated and carers informed. She also has some cognitive problems so we monitor her activity to check she is not leaving the house at unusual times. This monitoring is also mediated via a call centre. This clearly needs to be a dependable system, if it goes wrong it can be a threat to life, but safety is not the only concern. Three components of dependability will be considered here: safety, privacy and integrity.

With a network of interconnected devices, designing for safety is not straightforward as the failure or unexpected behaviour of one component can affect the whole system. With a socio-technical system the components 
include technology and humans, that is Mavis, the call centre, the carers and so on. Dependable systems detect errors before they lead to catastrophic failures. Humans are good at detecting and repairing failures. If, for example, the central locking system fails and Mavis is locked out, she should be able to contact the call centre and get emergency help. However, it is not enough to assume that because there is human supervision of the system all will be well. A case must be made for each possible error and the failures that could result. Technology designers often have a poor understanding of how people function in a socio-technical system and need help in doing this safety analysis.

Privacy is an important issue in the home. Mavis may be willing to give away some privacy in order to maintain her independence on the basis that the privacy she would lose should she have to move to an institution would be much greater. However, she needs to understand the precise nature of the transaction she is making. For example, people are very resistant to having cameras in their homes. This may be quite unreasonable if the camera is simply acting as a sophisticated movement detector. Of course, if the video images are transmitted outside of the home, their fears may be justified. Mavis needs to understand, and agree to who has access to the monitoring data collected and what these people know about her from it. She is then in a position to make an informed decision about whether the privacy given away is worth the independence gained.

Integrity is defined as security from external threats. If your door locks are controlled by a wireless link there is the possibility that a burglar with a scanner could obtain entry to your home. Could a person with malicious intent monitor the data being sent to the call centre to determine when Mavis is out of the house? In the early days of remote central locking for cars, thieves managed to steal cars in this way. More sophisticated encryption techniques were devised and the problem is now solved. The purpose of dependability analysis is to identify threats to the integrity of a system before they occur rather than after the event as in the above example.

Dependability of human-computer systems is a relatively new area for HCI (but see the work of Harrison and colleagues, for example, Pocock, Harrison, Wright \& Johnson, 2001). By breaking it down into component issues we can start to see how progress can be made. Dependability is an area where analysis is more important than empirical work. We cannot build alternative systems and see which kills the least people! The application of smart home technology to support older and disabled people is new, even though the technology itself may be old. There is much work to be done before we understand the problem well enough to specify methodologies or guidelines.

\section{Conclusion}

The two design problems considered here, the Virtual Pub and Mavis' smart home, illustrate how restricted the view of usability as ease-oflearning, ease-of-use and task fit is. The additional concepts suggested here are summarised in Table 3 along with some of the components of these concepts discussed. These all need further development and some may not stand the test of time. In this connection the history of the development of 
current conceptions of usability may be useful. This sees guidelines, representations and methodologies as the ultimate goals of usability engineering. It suggests empirical and analytic processes for achieving those goals.

Conventional conceptions of usability

Ease-of-learning

Low level ease-of-use

Task fit

Enjoyment

High engagement (as an individual or as a group)

Aesthetic attraction

Narrative completeness

Effective communication

Interpersonal awareness

Fluent turn allocation

Deixis

Dependability

Safety

Privacy

Integrity / security

Table 3. Components of usability identified in this paper.

At the beginning of this paper it was suggested that the home can act as a stimulus to change our ideas about usability, that our conception of usability must be broadened in other design contexts as well. Fun is not restricted to the home. Educational software needs to engender a high level of personal engagement for example. All the components of effective communication: interpersonal awareness, fluent turn allocation and deixis, apply in the workplace just as well as the home. Dependability has always been an issue in safety critical work contexts and integrity and privacy are rapidly becoming major issues in office technology. It is not overstating the case to say that a broader view of usability is crucial if HCI is to continue to successfully represent the needs of the user.

\section{References}

Checkland, P. \& Scholes, J. (1990). Soft systems methodology in action. Chichester: Wiley.

Clark, H. H. (1996). Using Language. Cambridge: CUP.

Csikszentmihalyi, M. \& Rathunde, K. (1993). The measurement of flow in everyday life: towards a theory of emergent motivation. Nebraska Symposium on Motivation, 40, 57-97.

DIRC, http://www.dirc.org.uk

Dix, A., Finlay, J., Abowd, G. \& Beale, R. (1998). Human-Computer Interaction. (2nd ed.) Hemel Hempstead: Prentice Hall.

Goffman, E. (1961). Fun in games. In E. Goffman (Eds.), Encounters: two studies in the sociology of interaction. Indianapolis: Bob Merril. 
Grice, H. P. (1957). Meaning. Philosophical Review, 66, 377-388.

Holtzblatt, K. \& Jones, S. (1993). Contextual inquiry: a participatory technique for system design. In A. Namioka \& D. Schuler (Eds.), Participatory design: principles and practice. Hillsdale, NJ: Erlbaum.

Hutchby, I. \& Wooffitt, R. (1998). Conversation Analysis. Principles practices and applications. Cambridge: Polity Press.

ISO 9241, ISO 13407, http://www.iso.ch

Microsoft (1995). The Windows interface guidelines for software design. Redmond: Microsoft Press.

Monk, A. F. (1998). Lightweight techniques to encourage innovative user interface design. In L. Wood (Eds.), User interface design: bridging the gap between user requirements and design. (pp. 109-129). Boca Raton: CRC Press.

Monk, A. F. (2000). User-centred design: the home use challenge. In A. Sloane \& F. van Rijn (Eds.), Home informatics and telematics: information technology and society. (pp. 181-190). Boston: Kluwer Academic Publishers.

Monk, A. F. \& Gale, C. (2002). A look is worth a thousand words: full gaze awareness in video-mediated conversation. Discourse Processes, $33,257-278$.

NASA TLX, http://iac.dtic.mil/hsiac/products/tlx/tlx.html

Pocock, S., Harrison, M., Wright, P. \& Johnson, P. (2001). THEA: a technique for human error assessment early in design. In M. Hirose (Eds.), Interact'01, Tokyo. (pp. 247-254). Amsterdam: IOS Press.

Short, J., Williams, E. \& Christie, B. (1976). The social psychology of telecommunications. London: John Wiley and Sons.

Sommerville, I. (2000). Software Engineering, 6th Edition. London: Pearson Education.

Vertegaal, R. (1999). The GAZE groupware system: mediating joint attention in multiparty communiation and collabroation. In M. G. Williams, M. W. Altom, K. Ehrlich \& W. Newman (Eds.), CHI'99, Pittsburgh, PA. (pp. 294-301). City: ACM Press.

Acknowledgement: I would like to thank all the members of the York Usability Research group, Jan Blom, Mark Blythe, Gordon Baxter, David Grayson, Darren Reed, Hokyoung Ryu, and Peter Wright who have contributed to this article through their original ideas and comments on drafts. Further information about the work of the group is available at www.yorkusabilityresearch.org. The author is supported by EPRC through Grant No. GR/M86446, and the DIRC project, also by PACCIT (award L328253006). 